PATRICIA GAUTHIER

ORCID: 0000-0001-5005-5227

Université de Poitiers, France

patricia.gauthier@univ-poitiers.fr

\title{
RÉAPPROPRIATION DU JANSÉNISME DANS QUELQUES ROMANS FRANÇAIS CONTEMPORAINS
}

L'histoire de Port-Royal est indissociable des tensions qu'a connues le monastère avec le pouvoir au XVII ${ }^{\mathrm{e}}$ siècle. La réforme voulue par Angélique Arnauld, qui rétablit la clôture en 1609 et met fin au relâchement qui régnait jusqu'à cette date, l'arrivée de directeurs spirituels comme saint François de Sales en 1619 et l'abbé de Saint-Cyran en 1635, vont créer les conditions d'une prospérité tant matérielle que spirituelle et intellectuelle. La communauté voit le nombre de ses religieuses augmenter. L'accueil de pensionnaires issues de la plus haute aristocratie ainsi que l'installation des Solitaires aux abords de l'abbaye en 1637-1638 contribuent au rayonnement de Port-Royal dans le siècle. Les Petites Écoles, créées en 1637 sous l'impulsion de Saint-Cyran, concurrencent avec un réel succès l'éducation alors majoritairement dispensée par les Jésuites. Le prestige du monastère est à son comble. Mais cette aura contient aussi les ferments de ce qui conduira à sa perte. Car si, au milieu des années 1650, l'affaire des cinq propositions sur la grâce contenues dans l'Augustinus de Jansénius fait entrer Port-Royal dans une crise ouverte dont le fondement doctrinal mènera au bras de fer avec Louis XIV, la présence de Saint-Cyran comme directeur spirituel avait déjà provoqué des heurts avec le pouvoir. Richelieu, méfiant à l'égard de cet ami de Jansénius dont il diffuse la pensée auprès des moniales, le fait emprisonner dès 1638. Le refus d'une quelconque subordination du religieux au politique au cœur de la pensée augustinienne, alors même que le roi de France est considéré comme un élu particulier de Dieu, cristallise le conflit. Louis XIV n'aura de cesse de mettre au pas cette 
communauté jugée d'autant plus dangereuse qu'elle recrute nombre de ses amis parmi d'anciens frondeurs (comme François de La Rochefoucauld, le cardinal de Retz ou le prince de Conti) et que les maîtres des Petites Écoles (comme Antoine Le Maître), les Solitaires (comme Robert Arnauld d'Andilly) et autres beaux amis de Port-Royal (comme Blaise Pascal) exercent un fort pouvoir d'attraction sur la vie intellectuelle du temps. Malgré une période d'accalmie lors de la Paix de l'Église (1668-1679) au cours de laquelle Louis XIV se fait même présenter Antoine Arnaud et Isaac Lemaistre de Sacy, les persécutions reprennent. La dispersion des religieuses en 1709 et la destruction du monastère de Port-Royal des Champs, ordonnée par le roi en 1710, en constituent la fin logique, sinon attendue, après les violences exercées contre les moniales dès 1661 (obligation de signer le formulaire condamnant Jansénius, envoi des principales religieuses en captivité $\left.^{1}\right)$. La démolition s'accompagne de l'exhumation de 3000 corps du cimetière de Port-Royal des Champs emmenés dans une fosse commune au cimetière de SaintLambert en 1711 et les murs mêmes de l'église sont détruits à la poudre en 1713.

Ce bref rappel d'épisodes bien connus de l'histoire de Port-Royal permet de mesurer combien la relation entre le bastion du jansénisme et le siècle a été conflictuelle. Au point que l'esprit de résistance des religieuses ait paradoxalement pu être considéré comme le ferment d'une déchristianisation au cours du $\mathrm{XVIII}^{\mathrm{e}}$ siècle menant à la Révolution ${ }^{2}$ et possiblement porteur d'un esprit laïc. On perçoit d'emblée la richesse mais aussi l'ambiguité liées au jansénisme dès lors qu'on se propose de le confronter à la notion de post-sécularité.

Richesse et ambiguïté inséparables également du lien qui unit ce courant religieux à la littérature. Jean Mesnard a montré que le paradoxe d'une austérité religieuse qui trouvait à s'exprimer dans une littérature qu'elle condamnait comme véhicule des passions n'était qu'apparent. Il montre ainsi les liens du milieu janséniste avec le monde des Lettres ${ }^{3}$. Saint-Cyran, qui a diffusé les idées jansénistes à Port-Royal, n'a jamais condamné la littérature. D'Andilly, quant à lui, a fréquenté assidûment le salon de Madame de Rambouillet, même s'il a choisi de mettre sa plume uniquement au service de la cause janséniste. L'intention religieuse justifie à ses yeux la pratique de l'art d'écrire. De même pour Le Maistre ou Le Maistre

${ }^{1}$ Pour un récit plus détaillé de l'histoire de Port-Royal, on consultera notamment : C.-A. SainteBeuve, Port-Royal, édition de Ph. Sellier, Robert Laffont, Paris 2004, qui reste une source incontournable, mais aussi : F. Hildesheimer, Le Jansénisme. L'Histoire et l'héritage, Desclée de Brouwer, Paris 1992 ; R. Taveneaux, Jansénisme et politique, Armand Colin, Paris 1965 ; M. Cottret, Histoire du jansénisme : XVII ${ }^{e}-X V I I I^{e}$ siècle, Perrin, Paris 2016 ; L. Plazenet, Port-Royal. Une anthologie, Flammarion, Paris 2012.

${ }^{2}$ Voir F. Hildesheimer, op. cit., chap. 4. Sur les liens possibles du jansénisme et de la laïcité, on se reportera aux actes à venir du colloque « Port-Royal et la République : 1940-1629 ? », colloque de la Société des Amis de Port-Royal, organisé par S. Icard, G. Métayer et L. Plazenet les 12 et 13 octobre 2017.

3 J. Mesnard, « Jansénisme et littérature », [dans :] Le Statut de la littérature. Mélanges offerts à Paul Bénichou, M. Fumaroli (dir.), Droz, Genève 1982, pp. 117-134. 
de Sacy. Pascal, lui, " engage le jansénisme sur la voie d'un destin littéraire ${ }^{4}$ avec les Provinciales puis les Pensées. Dans la polémique des lettres du Chevalier de Montalte, Mesnard souligne que la littérature se faisait nécessaire à l'existence même du jansénisme. Un rapport identique entre le religieux et la littérature se manifeste aussi bien chez P. Nicole que chez Fr. de La Rochefoucauld, traduisant un glissement de la spiritualité vers la morale auquel l'élite cultivée qui se tourne alors vers Port-Royal est sensible. Le milieu proche du jansénisme est de fait « un milieu où naturellement se développait la vie littéraire ${ }^{5}$ ». Mais, s'il est vrai que des pans entiers de la littérature de la deuxième moitié du XVII ${ }^{\mathrm{e}}$ siècle ont pu être influencés par une pensée augustinienne ${ }^{6}$ dont le jansénisme est un versant particulièrement rigoureux, on ne peut recenser de jansénistes, religieuses ou simples amis laïcs, qui soient explicitement entrés en littérature au XVII ${ }^{\mathrm{e}}$ siècle sous forme de personnages fictifs ou même que Port-Royal ait pu constituer le lieu où se serait développé tel ou tel récit fictif 7 .

Pourtant c'est bien le monastère lui-même, ses religieuses, ses proches, tout autant que l'esprit du jansénisme qui vont se retrouver dans des romans. Comme l'a montré Laurence Plazenet dans une étude de 2006 qui fait le bilan de la présence de Port-Royal dans la fiction romanesque du XVII ${ }^{\mathrm{e}}$ siècle jusqu'en 2004, cette présence court à travers les siècles et s'intensifie à la fin du $\mathrm{XX}^{\mathrm{e}}$ siècle $^{8}$. Elle mentionne notamment certaines œuvres de Pascal Quignard (Tous les Matins du monde) ou de Lydie Salvayre (La Puissance des mouches) mais aussi d'Erik Orsenna (Grand Amour) ou Gabriel Matzneff (Ivre du Vin perdu ou Maîtres et Complices), ainsi qu'un roman didactico-policier, Mystère Pascal ou la Mort du Père Noël de Frédéric Serror. La tendance n'a cessé d'être marquée depuis cette date et l'on mentionnera, sans prétendre à l'exhaustivité : Le Désert de la Grâce de Claude Pujade-Renaud (2007), Intrigue à Versailles d'Adrien Goetz (2009), Titus n'aimait pas Bérénice de Nathalie Azoulai (2015), mais aussi un roman de Laurence Plazenet elle-même, La Blessure et la Soif ${ }^{9}$ (2009). Comment expliquer l'intérêt des romanciers contemporains pour le jansénisme dans une société bien éloignée des débats théologiques ? Faut-il y voir la manifestation de la

${ }^{4}$ Ibidem, p. 126.

${ }^{5}$ Ibidem, p. 134.

${ }^{6} \mathrm{Qu}$ 'on songe par exemple aux lectures augustiniennes de La Princesse de Clèves ou du théâtre de J. Racine.

${ }^{7}$ On notera l'exception de la Clélie de Mademoiselle de Scudéry, qui présente un portrait de Timante dont le modèle est Robert Arnauld d'Andilly.

${ }^{8}$ L. Plazenet, « Port-Royal au prisme du roman (1657-2004) », RHLF 4, 2006, vol. 106, $<$ https://www.cairn.info/article_p.php ?ID_ARTICLE=RHLF_064_0927> [consulté le 26.07.2018]. L. Plazenet note qu'à l'exception de la Clélie, des Mémoires et aventures d'un homme de qualité et de L'Ingénu, « l'évocation de Port-Royal dans le roman est un fait postérieur à la parution de PortRoyal (1840-1859) de Sainte-Beuve » (L. Plazenet, « Port-Royal au prisme du roman... », p. 6).

${ }^{9}$ On ajoutera, de la même auteure : Disproportion de l'Homme (2010), qui renvoie de façon indirecte à Port-Royal par l'évidente référence à Pascal de son titre. 
post-sécularité comme une trace d'un éventuel retour du religieux d'autant plus intéressant que le jansénisme avait maille à partir avec le pouvoir temporel en son temps? Sa présence dans des romans permettrait ainsi une transposition des questions de l'époque à un contexte actuel propre à éclairer la post-sécularité contemporaine. Encore conviendrait-il de s'entendre sur le sens même de post-sécularité. Alain Badiou a interrogé la notion de siècle ${ }^{10}$ et l'on peut en effet se demander s'il est pertinent de considérer sur un même plan des romans parus à des dates qu'un événement souvent pris comme repère dans le retour du religieux, le 11 septembre 2001, sépare. Mais le retour du religieux est-il lui-même un phénomène pertinent dans la définition de la post-sécularité ? Clairement non pour plusieurs penseurs de cette notion, dont Badiou ${ }^{11}$ ou Jean-Luc Nancy:

Le « retour du religieux » dont on parle tant, et qui désigne un phénomène réel, ne mérite pas plus d'attention qu'aucun autre « retour ». Dans les phénomènes de répétition, de reprise, de relance ou de revenance, ce qui compte n'est jamais l'identique mais le différent. Car l'identique perd d'emblée son identité dans son propre retour, et la question devrait bien plutôt se poser à nouveaux frais, de savoir ce que la « sécularisation peut désigner et désigne inévitablement d'autre qu'un simple transfert de l'identique $»^{12}$.

Il apparaît donc difficile d'utiliser un événement ancré dans la chronologie pour doter un phénomène, observable de manière diffuse dans la société, d'un point $a b$ quo à la lumière duquel tout prendrait sens. D'autant qu'il faudrait se mettre d'accord sur la nature du religieux convoqué dans cette tentative. De quelle religion parle-t-on ? Et de son retour dans quel espace ? Du christianisme ? Du monothéisme en tant que religion du Livre ? En Europe ? Et si oui, que faire de la laïcité dite «à la française » ? On voit mal les romanciers répondre à ces questions de façon directe dans leur fiction à moins d'en faire des pensums. Le roman, dans les exemples précédemment cités, qu'il soit policier (Serror, Goetz, Salvayre), historique (Quignard, Pujade-Renaud, Plazenet et, pour partie, Azoulai) ou autobiographique (Orsenna) ne se présente pas comme porteur d'un message pour défendre une thèse. Aucune volonté chez ces romanciers de Port-Royal de revendiquer un usage, une place particulière de la religion dans la société d'aujourd'hui via leur récit. En termes de méthode, il faut donc se tourner vers autre chose que le placage d'une définition de la post-sécularité sur le matériau romanesque qui serait comme la négation de sa singularité fictionnelle et littéraire. Puisque Port-Royal a, on l'a dit, intrinsèquement à voir avec la littérature et avec un positionnement vis-à-vis du pouvoir, il faudrait donc plutôt se demander en quoi la récurrence de ce motif dans le roman définit un topos littéraire à même de dire quelque chose non pas du religieux, mais de ce que porte (apporte ?) la référence à cette religion-là dans notre société actuelle. Qu'en ont retenu les auteurs qui nous permette de mesurer l'écart entre ce que fut Port-Royal en son siècle et ce

\footnotetext{
10 A. Badiou, Le Siècle, Seuil, Paris 2005.

11 Voir ibidem, p. 233 notamment.

12 J.-L. Nancy, Déconstruction du christianisme, t. 1: La Déclosion, Galilée, Paris 2005, p. 9.
} 
que les romanciers en gardent aujourd'hui ? En d'autres termes, on se propose d'observer les variations dans le traitement de la présence du jansénisme au sein de quelques-unes des œuvres mentionnées plus haut, corpus restreint que nous impose le format du présent article : Tous les Matins du monde, La Puissance des mouches et Le Désert de la Grâce ${ }^{13}$. Il ne s'agira pas de penser le roman à l'aune de la post-sécularité. On abandonnera donc l'idée d'un roman illustrant la post-sécularité. On s'évertuera plutôt à chercher ce que notre siècle dit du siècle dans sa dimension profane (le romanesque) et religieuse (le jansénisme), ce qu'il renouvelle de leur rencontre pour penser la différence, sans préjuger de ce qu'une telle rencontre pourrait avoir de fécond dans l'approche de la post-sécularité.

\section{DE LA RÉSISTANCE}

Qu'il s'agisse de Tous les Matins du monde, de La Puissance des mouches ou du Désert de la Grâce, ces œuvres présentent un point commun : elles évoquent des personnages capables de résister aux pressions du monde extérieur. C'est le cas du compositeur Sainte Colombe que le lecteur découvre dans son désir constant de faire sécession. Dès le chapitre deux sont évoqués deux traits fondateurs de sa personnalité, qui ancrent son portrait dans une cohérence conforme à ce que le lecteur peut connaître des tensions entre Port-Royal et la cour. D'un côté, et à quelques lignes d'intervalle, il fréquente Lancelot, célèbre grammairien de Port-Royal, de l'autre « il avait été présenté au feu roi dans sa jeunesse et de ce jour, sans qu'on sût pourquoi, n'avait plus mis les pieds au Louvre ni au château-vieux de Saint-Germain » (TMM, pp.10-11). Fidèle à lui-même, il refuse ensuite l'ordre de Louis XIV de venir jouer à la cour tandis qu'il accepte l'invitation des jansénistes :

D'un côté les Libertins étaient tourmentés, de l'autre les Messieurs de Port-Royal étaient en fuite. Ceux-ci avaient eu le projet d'acheter une île en Amérique et de s'y établir comme les Puritains persécutés l'avaient fait. Monsieur de Sainte Colombe avait conservé des liens d'amitié avec Monsieur de Bures. Monsieur Coustel disait que les Solitaires poussaient l'excès d'humiliation au point qu'ils préféraient le mot monsieur au mot même de saint. Rue Saint-Dominique-d'Enfer, les enfants aussi se disaient entre eux « Monsieur » et ils ne se tutoyaient pas. Parfois un de ces Messieurs lui faisait envoyer un carrosse pour qu'il vînt jouer pour la mort d'un des leurs ou pour les Ténèbres. (TMM, p. 51)

L'usage de « Monsieur » entre les Solitaires fait l'objet d'un commentaire identique dans Sur l'idée d'une Communauté de Solitaires:

13 P. Quignard, Tous les Matins du monde, Gallimard, Paris 1990 (TMM) ; L. Salvayre, La Puissance des mouches, Seuil, Paris 1995 (PM) ; Cl. Pujade-Renaud, Le Désert de la Grâce, Actes Sud, Paris 2007 (DG). Les citations seront tirées des éditions de poche, respectivement : Folio plus classiques, Gallimard, Paris 2010 (TMM) ; Collection Points, Seuil, Paris 1997 (PM) ; et Babel, Actes Sud, Paris 2009 (DG). 
[Les Solitaires] ne renoncèrent pas à l'usage de la politesse mondaine. Ils utilisaient le mot «monsieur » pour se parler entre eux et même pour s'adresser aux enfants qu'ils instruisaient. Ils disaient « monsieur » à tout comme Saint François disait " frères » aux oiseaux et aux fils urticants des orties et au nuage qui passe et au soleil qui se lève. Ils ne se guidaient sur aucune règle extérieure, n'obéissaient à personne, jaloux seulement de leur retrait du monde, grands ménagers — grands aménageurs, grands draineurs de marécage — de leur retraite sauvage, grands jardiniers de leur silence. Ils étudiaient ${ }^{14}$.

Quignard souligne explicitement que l'acceptation de la politesse mondaine n'a rien d'une quelconque compromission et que la liberté reste première. À la phrase « [Ils] n'obéissaient à personne » répond le constat selon lequel « Le roi était mécontent de ne pas posséder Monsieur de Sainte Colombe » (TMM, p. 20). Ainsi se tisse un réseau sémantique qui associe la rupture avec le social à la préservation farouche de la liberté de pensée. On notera que les Libertins sont mis sur le même plan que les jansénistes par le romancier. La volonté de donner à l'étude un espace pour s'épanouir en dehors de toute contrainte renvoie à l'univers de Port-Royal par l'allusion aux Solitaires mais s'étend au-delà du domaine de la pensée religieuse ou de la foi par la référence aux Libertins. De manière subreptice, l'athéisme surgit à l'arrière-plan du roman comme point de fuite de l'indépendance revendiquée non plus par le seul personnage de Sainte Colombe mais par Quignard lui-même commentant le statut des Solitaires ${ }^{15}$.

Le Désert de la Grâce offre lui aussi une image de la résistance du milieu janséniste au pouvoir royal. Pujade-Renaud écrit un roman choral faisant alterner les points de vue de différents témoins de la destruction du monastère. Se succèdent les voix de Denis Dodart, médecin à Port-Royal, de Marie-Catherine Racine, fille du dramaturge, de Françoise de Joncoux, «l'Invisible » qui recueillit de très nombreux documents du mouvement janséniste, traductrice, copiste infatigable et soutien indéfectible des religieuses, de la Comtesse de Gramont, une des « belles amies » de Port-Royal, de Denise Le Petit, fille du libraire Camusat qui imprima certaines provinciales et de bien d'autres acteurs de la vie du milieu janséniste. Les différentes voix sont rassemblées en neuf tableaux, le premier, intitulé « Chiens », ouvrant le roman sur l'exhumation des cadavres du cimetière de Port-Royal à l'hiver 1711, le dernier, «Pèlerinages ", le faisant s'achever en 1719 par la visite aux ruines de Marie-Catherine Racine et ses filles. Alors que les fillettes jouent « à la vêture », leur mère se demande qui pourra un jour écrire la vie de Jean Racine. Elle plonge alors dans une rêverie portée par la poésie du lieu :

${ }^{14}$ P. Quignard, Sur l'idée d'une Communauté de Solitaires, Arléa, Paris 2015, p. 29.

15 Le personnage de Sainte Colombe est, de façon évidente, l'image même de cette capacité à rompre avec le social à laquelle Quignard a personnellement sacrifié en quittant ses fonctions chez Gallimard en 1994 et en se retirant à Sens. Il n'a de cesse de revenir sur ce thème comme on le voit encore par exemple dans Performances de Ténèbres ou dans Villa Amalia, dont l'héroïne est une sorte de double féminin contemporain du compositeur baroque. 
Le secret des sous-bois, le secret des consciences, impénétrables. Sur ce dernier point, les amis de Port-Royal avaient tenu bon, ni le roi ni le pape ne s'interposeraient par leurs décrets entre eux et Dieu. Ils l'avaient payé cher. A présent, Mme de Joncoux était morte. Et là-bas, dans son exil d'Amsterdam, Pasquier Quesnel atteignait ses quatre-vingt cinq ans. Quant aux membres du clergé qui avaient protesté contre la bulle pontificale, ils avaient été excommuniés. La fin d'une époque, d'une longue lutte ? Cependant, M. de Moramber demeurait confiant : le courant augustinien et gallican persisterait, souterrain mais efficace. (DG, pp. 280-281)

La dernière page du roman salue la persistance du mouvement en l'associant explicitement à la capacité de résistance des jansénistes. La peinture des «pèlerinages » laisse augurer d'une vivacité dans le temps dont la portée religieuse ne s'éteindra pas. C'est l'esprit de Port-Royal qui perdure, touché par la Grâce, dernier mot du roman, dont les accents ne sont pas sans ironie. Solidement documentée, l'œuvre de Pujade-Renaud est un hymne à la gloire de Port-Royal. Dans la polyphonie qu'elle met en musique, quasiment aucune place pour la voix des opposants aux jansénistes, si ce n'est celle de la Champmeslé, aigrie d'avoir été délaissée par Racine (DG, pp. 137-141) et celle de Mme de Maintenon. Encore ne s'exprime-t-elle que dans deux courts chapitres (DG, pp. 68 et 248). Formellement étouffée. Comme si rien ne pouvait s'opposer au réseau janséniste, à même de résister par la clandestinité ou le départ à l'étranger, au-delà de la destruction du monastère et de la violence exercée contre ses religieuses et ses fidèles.

Salvayre illustre à sa façon cette pérennité s'accomplissant dans le pèlerinage aux champs, tel qu'il a lieu à la fin du $\mathrm{XX}^{\mathrm{e}}$ siècle sous la houlette d'un guide de Port-Royal ${ }^{16}$. Car le pèlerinage aux ruines est florissant dans ces années 1990. Mais il n'a plus grand-chose à voir avec la Grâce. Le guide, narrateur de La Puissance des mouches, conduit en effet les touristes entre ruines et reliques conservées au musée de l'abbaye. Si, comme le fait remarquer Plazenet, Salvayre nomme la plupart des Solitaires et des Messieurs, c'est Pascal qui se détache :

Dès le début du chapitre 2, le narrateur explique qu'il organise la visite du musée autour du moment fort où le visiteur découvre la ceinture à clous de l'auteur des Pensées. Le masque mortuaire de Pascal suscite plusieurs développements. Le protagoniste, d'autre part, ne cesse de lire les Pensées, de les méditer, les commenter. Il consulte aussi la Correspondance. L'intérêt qu'il porte à Pascal l'engage même à faire des lectures érudites : il énumère les travaux d'Albert Béguin, la Vie de M. Pascal de Gilberte Périer, Sainte-Beuve, Léon Brunschvig, Henri Lefevre, Louis Lafuma, Georges Brunet, Lucien Goldman et Jean Mesnard ${ }^{17}$.

Située au centre du roman (au chapitre 9 sur un total de 21), et le constituant à elle seule, cette phrase : «Faut-il considérer, monsieur Jean, la lecture de Pascal comme un divertissement? » (PM, p. 77). Cette question centrale invite à reconsidérer le rapport qu'entretient le personnage avec l'auteur des Pensées. « Pascal le fascine parce qu'il met au grand jour l'absurdité de la condition humaine [...]. À

${ }^{16}$ Le lecteur découvre ce guide par la confession qu'il livre à son juge, à son médecin psychiatre et à l'infirmier de sa prison ainsi qu'à son avocat. On comprend peu à peu, comme dans une enquête policière, qu'il a tué son père.

${ }^{17}$ L. Plazenet, «Port-Royal au prisme du roman ... », p. 11. 
la lecture de Pascal, le héros du roman sort de la prison de la haine qu'il éprouvait [pour son père et qui l'a conduit à le tuer], échappe à "la puissance des mouches", pour commencer à penser et se découvrir lui-même ${ }^{18}{ }^{18}$. Plazenet ajoute que « Port-Royal est l'antithèse de la Germanité, bêtise et brutalité complaisantes par excellence ${ }^{19}$. À la lourdeur des hordes de touristes dont les Allemands sont le symbole aux yeux du narrateur ${ }^{20}$, le héros oppose « une leçon de vie et d'insoumission $»^{21}$. «Après bien des recherches et des atermoiements » (PM, p. 165), il quitte sa femme mais échoue dans sa nouvelle vie. Sans ressources, il se voit contraint de se rendre chez son père, qu'il tue après toute une enfance à l'avoir vu frapper sa mère, accomplissant dans son esprit œuvre de justice. La réponse à la question du chapitre neuf est donc négative. Il ne s'agit pas d'un divertissement au sens galvaudé du terme mais au contraire de l'occasion à saisir d'un véritable retour sur soi, de résister à l'enfermement d'une routine sociale (celle du mariage, celle du travail) pour s'affranchir de son carcan, quitte à briser les règles du pacte social :

Aujourd'hui donc que mon destin m'a séquestré entre ces quatre murs, j'incline à penser, monsieur Jean, que tout le malheur de l'homme vient au contraire de ce qu'il reste enfermé, enfermé dans le giron de ses mères, enferré jusqu'à l'âme dans sa passion de s'abêtir, détenu volontaire dans ses petites prisons portatives et dans d'autres plus vastes qu'il partage avec son bétail. (PM, pp. 119-120)

Salvayre se réapproprie donc le jansénisme par une lecture iconoclaste des Pensées, libérée de l'enjeu du pari et de la présence, cachée ou non, de Dieu. Pascal incarne ainsi une forme de résistance à l'emprise sociale, dont Descartes, malgré sa méthode et la mise du monde en coupe réglée par le bon sens, ne parvient pas à limiter la force, comme on le mesure à la lecture de La Méthode Mila ${ }^{22}$. Les deux romans forment un diptyque où s'inscrit en filigrane le jugement que Pascal porte sur le modèle philosophique cartésien : « inutile et incertain et pénible $»^{23}$.

Effacement du religieux et plaidoyer pour la tolérance

Dans ces exemples, y compris lorsque l'intrigue se déroule au XVII ${ }^{\mathrm{e}}$ siècle, les romanciers se réapproprient le jansénisme dans une vision qui privilégie sa puissance symbolique bien avant sa spécificité doctrinale. Les querelles autour de la grâce ne font pas l'objet de débats. C'est la force de résistance du mouvement

${ }^{18}$ Ibidem, p. 17.

19 Ibidem.

${ }^{20}$ On se reportera également à la description d'une visite où s'égrènent les remarques affligeantes des touristes (p. 57 en particulier). Ou encore le fait que lors de ces visites touristiques, le psittacisme guette le guide (cf. p. 120).

${ }^{21}$ Ibidem.

${ }^{22}$ Voir L. Salvayre, La Méthode Mila, Seuil, Paris 2005. Dans ce roman, Salvayre met à mal la philosophie de Descartes par l'intermédiaire d'un personnage aux prises avec sa mère vieillissante et grabataire, dont il devrait s'occuper en fils exemplaire et qui cherche en Descartes une aide pour supporter la mort annoncée. Mais c'est auprès de Mila, personnage de voyante libre et fantaisiste, qui n'a que faire de dénombrer et diviser les difficultés, qu'il va la trouver et reprendre goût à la vie.

${ }^{23}$ B. Pascal, Pensées, édition de Louis Lafuma, Seuil, Paris 1962, fragment 84. 
aux persécutions du pouvoir qui retient d'abord l'attention. Littéralement dans un premier temps, puis comme point de départ d'un élargissement de la résistance à toute forme de pression. C'est ainsi que la question doctrinale disparaît sous le thème de la possibilité pour l'individu de vivre librement sa foi ou son athéisme. La dimension proprement religieuse s'évanouit dès lors que l'intrigue s'installe dans la société du $\mathrm{XX}^{\mathrm{e}}$ siècle. De la lecture de Pascal, le personnage de Salvayre retient pour l'appliquer à son propre cas une réflexion morale et philosophique, mais pas religieuse. Ces exemples restent évidemment trop peu nombreux pour qu'on puisse en tirer des conclusions quant à la déclinaison de ce thème sous la forme d'un topos. Il trouve néanmoins un prolongement dans une véritable fascination pour le motif de la persécution elle-même. C'est notamment le cas chez Pujade-Renaud : l'exhumation des corps est évoquée à plusieurs reprises dans le roman, avec une insistance particulière sur les détails macabres ${ }^{24}$. Faut-il voir là une résonance avec la préoccupation des sociétés modernes à vouloir protéger la conscience individuelle et la liberté de croyance en insistant sur la violence liée à l'intolérance du pouvoir ? Les variations romanesques sur le jansénisme dans les œuvres qui nous occupent correspondent-elles à l'expression d'une post-sécularité ? La présence du jansénisme interrogeant le rapport de la religion au pouvoir absolu sous Louis XIV dans la littérature contemporaine s'exprimant au sein d'une démocratie pose une question caractéristique de la réflexion post-séculière. La réhabilitation du jansénisme comme force de résistance et la condamnation latente des persécutions afférentes plaident pour une sécularité apte à accueillir toutes les modalités de la foi et semble s'adresser à un lecteur acquis à une liberté de conscience libérée du joug du pouvoir. Les trois romans étudiés paraissent donc en phase avec l'idée de sécularité, dans l'acception de séculier comme étant « la condition moderne d'une raison autonome affranchie de la religion et qui assume la séparation de la foi et du savoir $»^{25}$. Mais dans le même temps, ces textes développent en filigrane, par contraste entre ce qu'ils dépeignent et la situation correspondant à la réalité du lecteur, l'idée que les démocraties ne constituent pas un espace propice à une vie spirituelle. Ils évoquent le jansénisme comme force d'engagement spirituel le plus haut permettant d'opposer cette exigence à une vie réduite à sa dimension trivialement sociale, sorte de « misère de l'homme sans $\mathrm{D} /$ dieu ». La méthode cartésienne pour conduire sa raison au sein d'une nature dont nous nous sommes rendus maîtres et possesseurs ne donne pas satisfaction au personnage de Salvayre ni au double féminin de Sainte Colombe dans

${ }^{24}$ Voir par exemple pp. $16-17$ ou pp. 73 et 114.

25 J.-M. Larouche, "Le sens des mots : postsécularisme et postséculière », SociologieS, [en ligne], Grands Résumés, Le Loup dans la bergerie. Le fondamentalisme chrétien à l'assaut de l'espace public, mis en ligne le 19 novembre 2013, <http://journals.openedition.org/sociologies/4527> [consulté le 19.06.2018], p. 4. 
Villa Amalia de Quignard ${ }^{26}$. Pourtant, le narrateur de La Puissance des mouches n'est pas préoccupé par la foi et, comme l'affirme Pierre Parlant, Quignard n'est pas plus chinois que janséniste ${ }^{27}$, ce que confirme la présence de l'athéisme dans l'évocation du jansénisme, même discrète.

Alors, pourquoi convoquer le jansénisme dans la fiction ? Est-ce à dire que Dieu n'est pas mort et que nos sociétés ne parviennent pas à se penser en dehors de toute référence à lui ? Faudrait-il alors tenter de transposer le nihilisme nietzschéen à l'expérience intérieure décrite par Georges Bataille ? Ou, pour le dire avec les mots de Nancy, faut-il en passer par la déclosion/déconstruction du christianisme ? Et à suivre ce chemin, peut-on imaginer que l'évocation romanesque du jansénisme soit un ferment paradoxal de cette déclosion?

\section{LITTÉRATURE ET DÉCLOSION}

En d'autres termes, peut-on et faut-il interroger la déclosion selon des modalités purement littéraires qui permettraient de penser la sécularité en dehors du christianisme ? En tout cas de s'extraire de son héritage et de l'envisager sous un angle non religieux, selon ce que Nancy nomme un "absenthéisme au-delà de toute position de croyance ou d'incroyance ${ }^{28}$ ? La présence du jansénisme dans les romans contemporains étudiés peut-elle être interprétée comme symptôme d'un corps perdu dans l'errance d'un impossible Dasein, clamant sa présence tout en niant sa définition en tant que principe ontologique ? Une différance au sens de Jacques Derrida, qui oblige à envisager la péremption du sens. Ce que la présence du jansénisme dans ces fictions contemporaines signale à défaut de le signifier relève d'un détachement ultime. La littérature donne à imaginer ce dépouillement de la signification en déniant à la Raison son rôle de mise en ordre et en forme du monde. Les romans ne pensent pas la sécularité et ne proposent pas d'éléments sur lesquels fonder une post-sécularité, car la raison n'est pas leur raison d'être. C'est ce que montre Salvayre en opposant Pascal à Descartes et, dans une moindre mesure, c'est ce qu'indique le dénouement du Désert de la Grâce, qui proclame la pérennité du jansénisme contre toute conclusion logique liée à la destruction du monastère. Car Pujade-Renaud entreprend avant tout d'écrire la légende de Port-Royal, faisant dire à Mlle de Théméricourt qu'il fallait « reprendre, célébrer — compléter peut-être — la geste de Port-Royal » et que « c'est à partir des textes qu'il faut la faire fructifier, cette mémoire, la transformer en légende,

${ }^{26}$ Le personnage principal de ce roman dont l'intrigue se passe aujourd'hui, Anne Hidden, est compositrice et décide un jour d'abandonner son métier, ses relations et sa vie tout entière. Rompant avec le social, elle trouve refuge à Ischia, comme Sainte Colombe s'isolait dans sa cabane. Voir P. Quignard, Villa Amalia, Gallimard, Paris 2006.

${ }_{27}$ P. Parlant, « Sino-miso-logie », Cahier Critique de Poésie 10, 2004/2, pp. 31-32.

28 J.-L. Nancy, op. cit., p. 133. 
parfois même au prix de quelque invention, [car] si l'imaginaire ne s'en mêle pas, les manuscrits cesseront de nous parler et mourront à leur tour » (DG, p. 228). L'imagination reine des facultés plutôt que maitresse d'erreur et de fausseté. C'est aussi ce que développe Quignard dans toute son œuvre. Le triomphe sur la pensée de ce qu'il nomme la Rêvée ${ }^{29}$ s'entend dans un espace littéraire comme lieu d'une refondation de soi en-dehors/au-delà du lien communautaire. Aussi s'intéresse-t-il à l'invention par Port-Royal d' « une communauté de solitaires » dont il souligne le caractère énigmatique ${ }^{30}$. Partant de l'idée « qu'à l'intérieur de tous les groupements humains il existe depuis toujours un désir de fuir qu'aucun groupe n'assume " ${ }^{31}$, il rêve, sur le modèle des Aufklärer de Spinoza, de " fonder un club antidémocratique fermé aux prêtres, aux magistrats, aux philosophes, aux politiques, aux éditorialistes, aux professeurs, aux galeristes $»^{32}$. Et il ajoute : «Il faut peut-être retourner à une diffusion plus solitaire et plus clandestine de l'œuvre d'art. Horror pleni, error pleni $»^{33}$. Refonder l'art pour libérer l'espace de la pensée. Rompre avec le social et, de facto, rendre possible une autre manière d'être au monde. «Enfin, dans cet espace, on respire. On ferme la bouche. On écrit. On est seul. On est soi. On respire $»^{34}$. Autre manière de dire que l'expérience individuelle ne saurait servir de modèle social reproductible mais se donnerait plutôt comme autre de la pensée du social, proposant une forme de déclosion. La littérature apparaît alors comme tremplin indispensable à la pensée du politique, non comme sa partie prenante mais comme force de remise en question intrinsèque. Pour Quignard, elle est fondamentalement dissidente et profondément corrosive. La littérature n'assigne pas le lecteur à la connaissance didactique qui impose un sens en contexte. Elle fait résonner en lui les signes de ce à quoi l'humain tente de ne pas renoncer.

L'évocation du jansénisme dans les romans étudiés n'invite pas plus à militer pour une ouverture sans réserve aux convictions religieuses qu'elle ne suggère d'accepter la religion comme expression d'un pluralisme communautaire induisant un pluralisme des formes de vie dans un élan de tolérance généralisée, ce qui correspondrait à une définition possible de la post-sécularité. Ces textes expriment plutôt la force de l'élan individuel, voire individualiste, désigné comme propre à la condition humaine. Ils donnent à voir l'homme comme animal social, mais animal social en rupture de groupe. À cette condition et contre l'idée de toute « communauté internationale ", l'écriture et l'art en général restent une arme de combat. Il ne s'agit donc pas de transposer les rapports du pouvoir et du jansénisme du

${ }^{29}$ « Je ne pense pas par arguments ; je pense toujours par images», écrit notamment P. Quignard dans Sur l'idée d'une Communauté de Solitaires, p. 13.

30 Ibidem, p. 28.

${ }^{31}$ Ibidem, p. 65.

${ }^{32}$ Ibidem, p. 67.

33 Ibidem.

${ }^{34}$ Ibidem, p. 66. 
$\mathrm{XVII}^{\mathrm{e}}$ au XXI ${ }^{\mathrm{e}}$ siècle. Mais de donner, par le romanesque, c'est-à-dire par l'image et non par le raisonnement, la Rêvée et non la Pensée, la possibilité d'appréhender, intuitivement et sans la formaliser, la place du vide dans nos sociétés. Sentir, à défaut de prouver, quelques 350 ans après les expériences de Pascal au Puy-deDôme, que le vide existe et qu'il est partie prenante des sociétés modernes dans leurs efforts pour comprendre la sécularité dans son devenir. Une post-sécularité libérée d'une référence obligée au religieux. Peut-être un vœu... pieux que le narrateur de La Puissance des mouches formule à sa manière : " La seule question qui m'intéresse $[\ldots]$ est celle-ci : comment prendre appui sur le néant ? » (PM, p. 159).

\section{JANSENISM REDUX IN SOME CONTEMPORARY FRENCH NOVELS}

\section{Summary}

The presence of Jansenism in a number of contemporary novels should lead to questioning the notion of post-secularity rather than illustrating it. The study of three of these novels (P. Quignard's Tous les Matins du monde, L. Salvayre's La Puissance des mouches and C. Pujade-Renaud's Le Désert de la Grâce) cannot be limited to interpreting them as heralds of a return to religion which some see as a defining feature of post-secularity while others deem it insufficient to define the notion. The analysis of the links between the royal authority and Port-Royal makes it possible to highlight the interest of novelists in the theme of persecution and the resistance of individuals to intolerance, while remaining at a distance from $17^{\text {th }}$ century theological debates. Beyond being a plea for freedom of conscience, these texts put it into perspective in a secularized democratic society that fails to conceive the place of the religious, or to conceive of itself outside that place. Literature thus shows the strength of fiction and art when it comes to considering man's place in the world.

Key words: Jansenism, Port-Royal, resistance, persecution, freedom of conscience, secularity, post-secularity, déclosion, literature. 